\title{
EnhanCED Data Driven Mode-Free AdAPTIVE YAW CONTROL OF UAV HELICOPTER
}

\author{
Dezhi $\mathrm{Xu}^{1}$ and Hongcheng Zhou ${ }^{2}$ \\ ${ }^{1}$ Key Laboratory of Advanced Control for Light Industry Processes, Ministry of \\ Education, Jiangnan University, Wuxi Jiangsu 214122, China \\ ${ }^{2}$ Institute of Information, JinLing Institute of Technology, Nanjing 211169, China
}

\begin{abstract}
An enhanced data driven model-free adaptive yaw control tracking control scheme is proposed for the yaw channel of an unmanned-aerial-vehicle (UAV) helicopter which is non-affine in the control input in this paper. Through dynamic linearization and observer techniques, the proposed control algorithm is only based on the pseudo-partial derivative (PPD) parameter estimation derived online from the I/O data of the yaw channel of an UAV helicopter, and Lyapunov-based stability analysis is utilized to prove all signals of close-loop control system are bounded. Compared with the traditional model free adaptive control (MFAC), the proposed enhanced MFAC algorithm can make the close-loop control system with stronger robustness and better anti-jamming ability. Finally, simulation results of the UAV yaw channel are offered to demonstrate the effectiveness of the proposed novel control technique.
\end{abstract}

\section{KEYWORDS}

Unmanned-aerial-vehicle helicopter, yaw control, model free adaptive control, internal model

\section{INTRODUCTION}

The potential use of unmanned-aerial-vehicle (UAV) helicopter can be applied in military and civilian, although military applications dominate the non-military ones. Military and civilian applications include power lines inspection, surveillance, national defense, agriculture, disaster rescue applications and so on [1]. Dynamics of UAV helicopter are strongly nonlinear, seriously multi-variable coupled, inherently unstable and a non-minimum phase system with time-varying parameters. So controlling the UAV helicopter is not an easy task. In the control area, to improve the performance of UAV helicopter has been an important focus [1-2].

As a highly nonlinear and uncertain system, helicopter flight control system design has been dominated by linear control techniques. In the past few decades, linear control algorithms have been extensively researched [1, 3-5]. Many linear control technologies were used to design the UAV helicopter control system [1, 6-10]. However, for the tracking control, the controller based on fixed linear models may result in an unacceptable response and even the instability of the closed-loop system. Because linearized models cannot guarantee the global model approximation, nonlinear control methods have been used in the control system design, such as [2, 11-12]. Furthermore, in a lot of control systems, the nonlinear model of plant dynamics is generally nonaffine in input and is commonly simplified around a trim point, that is, an operating point is dependent on the current system states [13]. Coupled with the uncertainties under the varying environment and the changing flight conditions, developing a controller to opportune compensate for the time varying uncertainties have been a more difficult task [14].

DOI : $10.5121 /$ ijics.2015.5402 
As one of the data-driven control methods, MFAC has been proposed and applied in several areas. MFAC algorithm based on compact form dynamic linearization (CFDL), partial form dynamic linearization (PFDL), and full form dynamic linearization (FFDL) have been proposed by Hou for single-input single-output (SISO), multi-input single-output (MISO), and multi-input multi-output (MIMO) systems [15-17]. However, the MFAC is still developing. How to prove the stability and convergence of the tracking problems is one of the open problems in MFAC [20]. We all know that the Lyapunov function is widely used to analyse the stability of close-loop systems [15].

In this paper, we focus on how to design a data-driven controller based on the Lyapunov method. Inspired by the work of dynamic linearization technique of Hou [15], we present an enhanced adaptive observer based on control strategies for nonlinear process systems in which the pseudopartial derivative (PPD) theory is used to dynamically linearize the nonlinear system. First, a novel adaptive strategy for computing the PPD term is designed by using the Lyapunov method. Then, the internal model approach is used to design the data-driven controller via CFDL. The stability analysis for tracking error of the proposed algorithm is provided. Last, an application of the proposed controller design for a small-scale UAV helicopter mounted on an experiment platform is also given to show the effectiveness of the control algorithm.

The rest of this paper is organized as follows. In Section 2, the yaw dynamic of the helicopter and the simplified model are given. In Section 3, the main results of internal model approach based on data-driven control via CFDL are proposed. Simulation results are presented to show the effectiveness of the proposed technique in Section 4. Finally, some conclusions are given at the end of this paper.

\section{Problem DeSCRIPTION}

It is clearly known that yaw channel control of controlling small scale UAV helicopters is one of the most challenging jobs $[4,10]$. Due to the small size of small-scale UAV helicopter, the torque combined with the yaw dynamic is highly sensitive. In order to improve the performance of the yaw control, a more precise channel model characterizing of UAV is necessary. A framework of the simulation model for the UAV helicopter (see Fig.1) is set up using rigid body equations of motion of the helicopter fuselage.

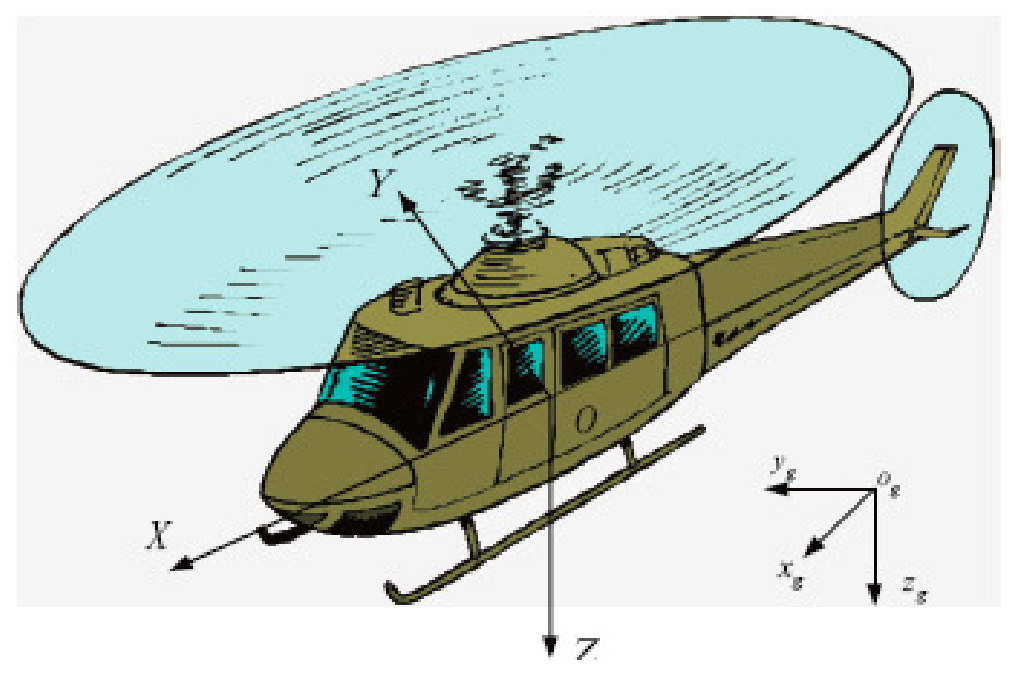

Figure 1: The frame of helicopter 
In this way the influence of the aerodynamic forces and moments working on the helicopter are expressed. The total aerodynamic forces and moments acting on a helicopter can be computed by summing up the contributions of all parts on the helicopter (including main rotor, fuselage, tail rotor, vertical fin and horizontal stabilizer). So, the yaw channel dynamic equations are given by:

$$
\begin{aligned}
& \dot{\varphi}=r \\
& I_{z z} \dot{r}=N_{m r}+N_{t r}+N_{f u s}+N_{h s}+N_{v f}
\end{aligned}
$$

where $\varphi$ and $r$ are the yaw angle and angular rate of the helicopter; $I_{z z}$ is the inertia around $\mathrm{z}$ axis; $N_{m r}, N_{t r}, N_{h s}, N_{f u s}$ and $N_{v f}$ present the torque of main rotor, tail rotor, horizontal, fuselage and vertical fin worked on the helicopter respectively.

In hovering and low-velocity flight, the dominant torque is caused by main rotor and tail rotor [18]. By simplifying the fuselage and vertical fin damping, the yaw channel dynamics can be rewritten as:

$$
\begin{aligned}
& \dot{\varphi}=r \\
& I_{z z} \dot{r}=-Q_{m r}+T_{t r} l_{t r}+b_{1} r+b_{2} \varphi
\end{aligned}
$$

where $Q_{m r}$ is the main rotor's torque, $T_{r}$ is the tail rotor's thrust, $l_{t r}$ is the distance between the tail rotor and z-axis, $b_{1}$ and $b_{2}$ are damping constants.

$$
\begin{aligned}
Q_{m r}= & \frac{1}{8} C_{d 2} \rho c \Omega^{2}\left(R^{4}-R_{0}^{4}\right) \theta_{m r}^{2} \\
& +\left[8 C_{d 2} \Omega \sqrt{\rho \pi R^{2}\left(2 C_{1} \theta_{m r}+C_{2}^{2}-C_{2} \sqrt{C_{2}^{2}+4 C_{2} \theta_{m r}}\right)}\left(R_{0}^{3}-R^{3}\right)\right. \\
& +4 a \Omega \sqrt{\rho \pi R^{2}\left(2 C_{1} \theta_{m r}+C_{2}^{2}-C_{2} \sqrt{C_{2}^{2}+4 C_{1} \theta_{m r}}\right)}\left(R^{3}-R_{0}^{3}\right)++6 C_{d 2} C_{1}\left(R^{2}-R_{0}^{2}\right) \\
& \left.+6 a C_{1}\left(R_{0}^{2}-R^{2}\right)+6 C_{d 1} \rho \pi \Omega^{2} R^{2}\left(R^{4}-R_{0}^{4}\right)\right] \frac{c \theta_{m r}}{48 \pi R^{2}}+\left[3 C_{d 2} C_{2} \sqrt{C_{2}^{2}+4 C_{1} \theta_{m r}}\left(R_{0}^{2}-R^{2}\right)\right. \\
& +3 a C_{2} \sqrt{C_{2}^{2}+4 C_{1} \theta}\left(R^{2}-R_{0}^{2}\right)+6 C_{d 0} \rho \pi \Omega^{2} R^{2}\left(R^{4}-R_{0}^{4}\right)+3 C_{d 2} C_{2}^{2}\left(R^{2}-R_{0}^{2}\right) \\
& \left.+4 C_{d 1} \Omega \sqrt{\rho \pi R^{2}\left(2 C_{1} \theta_{m r}+C_{2}^{2}-C_{2} \sqrt{C_{2}^{2}+4 C_{1} \theta_{m r}}\right)}\left(R_{0}^{3}-R^{3}\right)+3 a C_{2}^{2}\left(R_{0}^{2}-R^{2}\right)\right] \frac{c}{48 \pi R^{2}}
\end{aligned}
$$

The brief description of the forces and torques computing can be given by using the blade element method [18]. The torque which is generated by main rotor can be described by:

$$
Q_{m r}=\int_{R_{0}}^{R}\left(\frac{\rho \Omega^{2} r^{2} C_{l} c \phi}{2}+\frac{\rho \Omega^{2} r^{2} C_{d} c}{2}\right) r d r
$$

with $\phi=v_{1} /(\Omega r), C_{l}=a \alpha, C_{d} \approx C_{d 0}+C_{d 1} \alpha+C_{d 2} \alpha^{2}$, where $\rho, a, r, \alpha, c, v_{1}, \phi$ and $\Omega$ are density of air, slope of the lift curve, speed radial distance, the angle of attack of the blade element, chord of the blade, induced speed, inflow angle and rotor speed of the main rotor respectively. After complete employment with the help of Maple, we obtain (4) with $C_{1}=\frac{1}{6} \rho a b c \Omega^{2}\left(R^{3}-R_{0}^{3}\right), C_{2}=\frac{1}{8} \rho a b c \Omega \sqrt{2 / \rho \pi R^{2}}\left(R^{2}-R_{0}^{2}\right)$, where $\theta_{m r}, R$ and $b$ are 
pitch angle of main rotor, radial and number of the rotor. Similarly, the force which is created by the tail rotor can be represented by the following form

$$
\begin{gathered}
T_{t r}=\frac{1}{2} \rho a_{t r} b_{t r} c_{t r} \Omega_{t r}^{2} \int_{R_{t r 0}}^{R_{t r}}\left(\theta_{t r} r_{t r}^{2}-\frac{v_{t r 1}}{\Omega_{t r}} r\right) d r_{t r} \\
v_{t r 1}=\sqrt{\frac{T_{t r}}{2 \rho A_{t r}}}
\end{gathered}
$$

Combing (5) with (6), we have

$$
\begin{aligned}
& T_{t r}=\frac{1}{2} \rho a_{t r} b_{t r} c_{t r} \Omega_{t r}^{2} \int_{R_{t r}}^{R_{t r}}\left(\theta_{t r} r_{t r}^{2}-\sqrt{\frac{T_{t r}}{2 \rho A_{t r}}} \frac{r}{\Omega_{t r}}\right) d r_{t r} \\
& =C_{3} \theta_{t r}+\frac{1}{2} C_{4}\left(C_{4}+\sqrt{C_{4}^{2}+4 C_{3} \theta_{t r}}\right)
\end{aligned}
$$

with $C_{3}=\frac{1}{6} \rho a_{t r} b_{t r} c_{t r} \Omega_{t r}^{2}\left(R_{t r}^{3}-R_{t r 0}^{3}\right), C_{4}=\frac{1}{8} \rho a_{t r} b_{t r} c_{t r} \Omega_{t r} \sqrt{2 / \rho \pi R_{t r}^{2}}\left(R_{t r}^{2}-R_{t r 0}^{2}\right)$. where $a_{t r}$, $c_{t r}, b_{t r}, \Omega_{t r}, \theta_{t r}, r_{t r}, v_{t r}$ and $A_{t r}$ are slope of the lift curve, chord of the blade, number of the rotor, speed of the tail rotor, pitch angle, radial distance, induced tail rotor's speed, and the tail rotor disc's area, respectively.

Similarly, the force of the main rotor is

$$
T_{m r}=C_{1} \theta_{m r}+\frac{1}{2} C_{2}\left(C_{2}+\sqrt{C_{2}^{2}+4 C_{1} \theta_{m r}}\right)
$$

The yaw angle $\varphi$ is controlled through the $\theta_{t r}$. The $\theta_{t r}$ is chosen as the control input $u$. The $\varphi$ is chosen as the control objective $y$. By above UAV yaw-channel modeling, we can see that it is difficult to design a model-based feedback controller to stabilization system (2). Moreover, the input output relation of UAV yaw-channel modeling can be written in the following Nonlinear Auto Regressive with eXogenous input (NARX) model:

$$
y(k+1)=f\left(y(k), \cdots, y\left(k-n_{d}\right), u(k), \cdots, u\left(k-n_{n}\right)\right)+d(t)
$$

where $d(t)$ denotes the external disturbance and assumes its slowly time-varying. Currently, in order to control the yaw-channel of UAV, various control methods are proposed by [6-9], for example, nonlinear adaptive control, backstepping control, and neural network control and so on. For the nonlinear system (9), there must exist a parameter $\vartheta(k)$, called PPD, system (9) can be transformed into the following CFDL description when $|\Delta u(k)| \neq 0$ :

$$
\Delta y(k+1)=\Delta u(k) \vartheta(k)+\Delta d(k)=\Phi^{T}(k) \theta(k)
$$

where $\Delta d(k)=d(k)-d(k-1), \theta(k)=[\vartheta(k), \Delta d(k)]^{T}, \Phi(k)=[\Delta u(k), 1]^{T}$. 


\section{Methodology}

\subsection{Model Parameter Estimation Algorithm}

The proposed parameter identification observer has the following structure

$$
\hat{y}(k+1)=\hat{y}(k)+\Phi^{T}(k) \hat{\theta}(k)+K e_{o}(k)
$$

where $e_{o}(k)=y(k)-\hat{y}(k)$ is the output estimation error, $\hat{\theta}(k)=[\hat{\vartheta}(k), \Delta \hat{d}(k)]^{T}$, and the gain $K$ is chosen such that $F=1-K$ in the unit circle.

Hence, in view of (10) and (11), the output estimation error dynamics is given by

$$
e_{o}(k+1)=\Phi^{T}(k) \tilde{\theta}(k)+F e_{o}(k)
$$

where $\tilde{\theta}(k)=\theta(k)-\hat{\theta}(k)$ represents the parameter estimation error. The adaptive update law for the estimated parameters $\theta(k)$ can be chosen as

$$
\hat{\theta}(k+1)=\hat{\theta}(k)+\Phi(k) \Gamma(k)\left(e_{o}(k+1)-F e_{o}(k)\right)
$$

The gain $\Gamma(k)$ is chosen as follows

$$
\Gamma(k)=2\left(\|\Phi(k)\|^{2}+\mu\right)^{-1}
$$

where $\mu$ is a positive constant, hence, $\Gamma(k)$ is positive definite for all $k$. Notice that, by virtue of assumption $\|\Phi(k)\| \leq \Omega, \Gamma(k)$ can be lower bounded as

$$
\|\Gamma(k)\| \geq \frac{2}{\Omega^{2}+\mu}=\gamma>0
$$

By taking into account (12) and (13), the estimation error dynamics can be written as

$$
\begin{aligned}
& e_{o}(k+1)=\Phi^{T}(k) \tilde{\theta}(k)+F e_{o}(k) \\
& \tilde{\theta}(k+1)=H \tilde{\theta}(k)
\end{aligned}
$$

where $H_{c}$ is given by $H=I_{2}-\Phi(k) \Gamma(k) \Phi^{T}(k)$ and $I_{2}$ denotes the $(2 \times 2)$ identity matrix.

Theorem 1: The equilibrium $\left[e_{o}, \tilde{\theta}^{T}\right]^{T}=\left[0, \mathbf{0}_{2 \times 1}^{T}\right]^{T}$ of the system (15) is globally uniformly stable. Furthermore, the estimation error $e_{o}(k)$ converges asymptotically to 0 .

Proof: Consider the Lyapunov function

$$
V_{1}(k)=P e_{o}^{2}(k)+\lambda \tilde{\theta}^{T}(k) \tilde{\theta}(k)
$$

where $\lambda, P$ are positive constants and $P$ is the solution by $P+F^{2} P=Q$ with $Q$ is positive constant. 
By taking into (15), we have

$$
\begin{aligned}
& \Delta V_{1}(k)=V_{1}(k+1)-V_{1}(k) \\
& =P \Phi^{T}(k) \tilde{\theta}(k) \tilde{\theta}^{T}(k) \Phi(k)+2 P F \Phi^{T}(k) \tilde{\theta}(k) e_{o}(k)+P e_{o}^{2}(k)+P F^{2} e_{o}^{2}(k) \\
& +\tilde{\theta}^{T}(k)\left(\lambda H^{T} H-\lambda\right) \tilde{\theta}(k)=-Q e_{o}^{2}(k)-\Theta^{T}(k)\left[\lambda \mu \Gamma^{T}(k) \Gamma(k)-P\right] \Theta(k)+2 P F e_{o}(k) \Theta(k) \\
& \leq-Q\left|e_{o}(k)\right|^{2}-\left[\lambda \mu \Gamma^{T}(k) \Gamma(k)-P\right]\left\|\left.\Theta(k)\right|^{2}+2 P F\left|e_{o}(k)\right|\right\| \Theta(k) \| \\
& \leq-c_{1}\left|e_{o}(k)\right|^{2}-c_{2} \|\left.\Theta(k)\right|^{2}
\end{aligned}
$$

Where $\Theta(k)=\Phi^{T}(k) \tilde{\theta}(k), \quad c_{1}=Q-\frac{1}{\varsigma}, \quad c_{2}=\mu \lambda \gamma^{2}-P-\varsigma P^{2} F^{2}$. Hence, $\Delta V_{1}(k) \leq 0$ provided that $\varsigma, Q$ and $\lambda$ satisfy the following inequalities

$$
Q>\frac{1}{\varsigma}, \mu \lambda \gamma^{2}-P-\varsigma P^{2} F^{2}>0
$$

Notice that $\Delta V_{1}(k)$ is negative definite in the variables $e_{o}(k), \Theta(k)$. Since $V(k)$ in a decreasing and non-negative function, it converges to a constant value $V_{1}^{\infty} \geq 0$, as $k \rightarrow \infty$, hence, $\Delta V_{1}(k) \rightarrow 0$. This implies that both $e_{o}(k)$ and $\tilde{\theta}(k)$ remain bounded for all $k$, and $\lim _{k \rightarrow \infty} e_{o}(k)=0$.

\subsection{Controller Design}

Based on the observer (11), the data-driven inverse control law can be described as

$$
\begin{aligned}
& u(k)=u(k-1)+\frac{\hat{\vartheta}(k)\left(y^{*}(k+1)-\hat{y}(k)-K e_{o}(k)-\Delta \hat{d}(k)\right)}{\hat{\vartheta}^{2}(k)+\alpha}, \text { for }|\Delta u(k)| \leq \delta \\
& u(k)=u(k-1)+\delta \operatorname{sign}(\Delta u(k)), \text { for }|\Delta u(k)|>\delta
\end{aligned}
$$

where $y^{*}(k)$ is reference trajectory. $\alpha$ and $\delta$ as given finite positive numbers. Notice that, in many practical systems, because their actuators cannot change too fast, the number $\delta$ can be obtained.

Define observer tracking error $e(k)=y^{*}(k)-\hat{y}(k)$, thus

$$
e(k+1)=y^{*}(k+1)-\hat{y}(k+1)=y^{*}(k+1)-\hat{y}(k)-\Phi^{T}(k) \hat{\theta}(k)-K e_{o}(k)
$$

The robustness of the stability and the performance for data-driven control law (16) are given in Theorem 2. 
Theorem 2: For given $\left|y^{*}(k)-y^{*}(k-1)\right| \leq \Delta y^{*}$, using the data-driven control law (16), the solution of close-loop observer error system (17) is uniformly ultimately bounded (UUB) [19] for all $k$ with ultimate bound $\lim _{k \rightarrow \infty}\left|e_{o}(k)\right| \leq \frac{a_{2}}{1-a_{1}}$, where $\Delta y^{*}$ is a given positive constant, $0<s_{0}(k) \leq 1$,

$$
\begin{aligned}
& a_{1}=1-s_{0}(k)+\frac{s_{0}(k) \alpha}{\hat{\vartheta}^{2}(k)+\alpha}, \\
& a_{2}=\left(1-s_{0}(k)+\frac{s_{0}(k) \alpha}{\hat{\vartheta}^{2}(k)+\alpha}\right)\left|\Delta y^{*}-K e_{o}(k)-\Delta \hat{d}(k)\right| .
\end{aligned}
$$

Proof: Define a variable $s_{0}(k)$ where $0<s_{0}(k) \leq 1$ for all $k$. The control law (16) is equivalently expressed as

$$
\Delta u(k)=\frac{\hat{\vartheta}(k)\left(y^{*}(k+1)-\hat{y}(k)-K e_{o}(k)-\Delta \hat{d}(k)\right)}{\hat{\vartheta}^{2}(k)+\alpha} s_{0}(k)
$$

where

$$
\begin{array}{ll}
s_{0}(k)=1, & \text { for }|\Delta u(k)| \leq \delta \\
0<s_{0}(k)<1, & \text { for }|\Delta u(k)|>\delta
\end{array}
$$

Using (18), (17) becomes

$$
\begin{aligned}
& |e(k+1)|=\left(1-s_{0}(k)+\frac{s_{0}(k) \alpha}{\hat{\vartheta}^{2}(k)+\alpha}\right) \times\left|\left(y^{*}(k+1)-\hat{y}(k)-K e_{o}(k)-\Delta \hat{d}(k)\right)\right| \\
& =\left(1-s_{0}(k)+\frac{s_{0}(k) \alpha}{\hat{\vartheta}^{2}(k)+\alpha}\right) \times\left|\left(y^{*}(k+1)-y^{*}(k)+e(k)-K e_{o}(k)-\Delta \hat{d}(k)\right)\right| \\
& \leq\left(1-s_{0}(k)+\frac{s_{0}(k) \alpha}{\hat{\vartheta}^{2}(k)+\alpha}\right)|e(k)|+\left(1-s_{0}(k)+\frac{s_{0}(k) \alpha}{\hat{\vartheta}^{2}(k)+\alpha}\right) \Delta y^{*}-K e_{o}(k)-\Delta \hat{d}(k) \\
& =a_{1}\left|e_{o}(k)\right|+a_{2}
\end{aligned}
$$

Choosing a Lyapunov function as $V(k)=\left|e_{o}(k)\right|$, from (19), one has $\Delta V(k+1)=\left|e_{o}(k+1)\right|-\left|e_{o}(k)\right|=\left(1-a_{1}\right) V(k)+a_{2}$. Since $0 \leq a_{1}<1$ and $a_{2}$ is bounded, according to the lemma in [19], using the control law (16), the results of close-loop observer system (17) are UUB for all $\mathrm{k}$ with ultimate bound $\lim _{k \rightarrow \infty}\left|e_{o}(k)\right| \leq \frac{a_{2}}{1-a_{1}}$.

Corollary 1: Under the controller (16), together with the observer (11), adaptive laws (13), we can guarantee that the system (9) tracking error $e_{c}(k)=y^{*}(k)-y(k)$ is UUB with ultimate bound $\lim _{k \rightarrow \infty}|e(k)| \leq \frac{a_{2}}{1-a_{1}}$. 
Proof: Since

$$
e_{c}(k)=e(k)-e_{o}(k)
$$

Taking the absolute value and limiting on both sides of (20), we obtain

$$
\lim _{k \rightarrow \infty}|e(k)| \leq \lim _{k \rightarrow \infty}\left|e_{o}(k)\right|+\lim _{k \rightarrow \infty}\left|e_{c}(k)\right| \leq \frac{a_{2}}{1-a_{1}}
$$

So the tracking error $e(k)$ is UUB for all $k$ with ultimate bound $\lim _{k \rightarrow \infty}|e(k)| \leq \frac{a_{2}}{1-a_{1}}$.

\subsection{Enhanced Controller Design}

In this paper, the internal model structure is adopted to improve the robustness of close-loop system. Where observer (11) is seem as internal model. Although adaptive internal model can ensure the close-loop is stable. The modeling errors still exists, and it will reduce the robustness and stability. The traditional method is to introduce a feedback low-pass filter. In order to further improve the robustness, the low-pass filter can be designed in the proposed controller. The block diagram of the enhanced MFACl method is shown in Fig. 2, where the low-pass filter is described as

$$
F(z)=\frac{1-\varsigma}{1-\varsigma z^{-1}}
$$

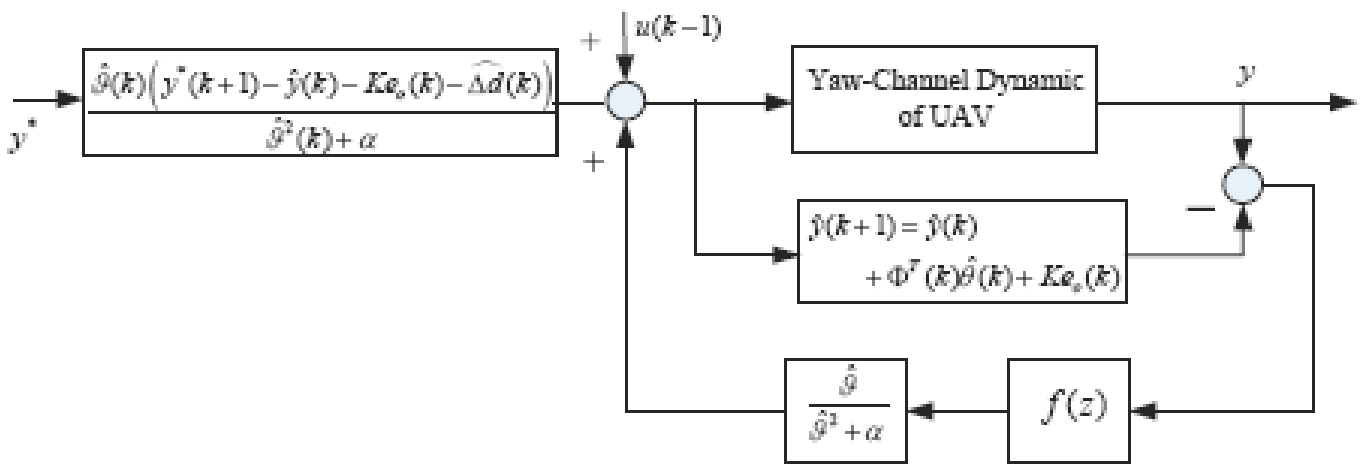

Figure 2: Block diagram of enhanced model free adaptive

Under the control architecture as shown in Fig. 2, the equivalent control law can be represented as follows:

$$
\begin{array}{r}
u(k)=u(k-1)+\frac{\hat{\vartheta}(k)\left(y^{*}(k+1)-\hat{y}(k)-K e_{o}(k)-\Delta \hat{d}(k)-F(z) e_{o}(k)\right)}{\hat{\vartheta}^{2}(k)+\alpha}, \\
\text { for }|\Delta u(k)| \leq \delta \\
u(k)=u(k-1)+\delta \operatorname{sign}(\Delta u(k)), \text { for }|\Delta u(k)|>\delta
\end{array}
$$

Corollary 2: For given $\left|y^{*}(k)-y^{*}(k-1)\right| \leq \Delta y^{*}$, using the enhanced model free control law (23), the solution of tracking error $e_{c}(k)$ is UUB where $\Delta y^{*}$ is a given positive constant.

Proof: The proof is similar as Theorem 2 with Corollary 1. 


\section{EXPERIMENTAL SETUP}

In this section, the control algorithm is validated by the simulation model which is obtained from the helicopter-on-arm platform [10]. First, the parameters of the non-affine nonlinear yaw dynamic model are identified as follows

$$
\begin{aligned}
& \dot{\varphi}=r \\
& \dot{r}=k_{1} r+k_{2} \varphi+k_{3} \theta_{t r}+k_{4} \theta_{t r}^{2}+k_{5} \Omega \theta_{t r}+d(t)
\end{aligned}
$$

with $k_{1}=-1.38, k_{2}=-3.33, k_{3}=63.09, k_{4}=11.65, k_{5}=-0.14$ and $\Omega=1200$. It is obviously that $k_{3} \theta_{t r}+k_{4} \theta_{t r}^{2}+k_{5} \Omega \theta_{t r}$ is a non-affine nonlinear function with respect to the control input $\theta_{t r}$.

For the proposed control law, we choose the sampling time $T_{s}=1$. The parameters of proposed control law in Section III are $k_{c}=0.9, \mu=0.1, \alpha=0.01, \delta=0.2, \grave{\mathrm{o}}=10^{-10}$ and $\hat{\phi}(1)=10$. The parameter of filter (22) is $\varsigma=0.75$.

In the following simulations, the initial conditions are $\varphi(0)=5, r(0)=0$. The tracking command of $\varphi_{c}$ is

$$
\varphi_{c}=\left\{\begin{array}{cr}
10, & t \leq 20 \\
15, & 20<t \leq 40 \\
25, & \mathrm{t}>40
\end{array}\right.
$$

Pass $\varphi_{c}$ through a filter, such as $F_{c}=\frac{\varphi_{d}}{\varphi_{c}}=\frac{0.8}{s+0.8}$. So desired trajectory $\varphi_{d}=y_{d}=\frac{0.8}{s+0.8} \varphi_{c}$. In order to verify the robustness of our proposed method for the unknown uncertainties/disturbances, in the simulation, the disturbance is designed to change according to the time-varying changing, i.e.

$$
d(t)=\left\{\begin{array}{ccc}
0 & \mathrm{deg} / s^{2} & 10 \geq \mathrm{t} \\
5 \sin (\pi t)+4 \cos (2 \pi t)+3 \cos (3 \pi t) \sin (2 \pi t) & \mathrm{deg} / s^{2} & 10<\mathrm{t}
\end{array}\right.
$$

We compare two control methods, they are proposed in [17] and in this paper. System responses are shown by the control method of [17] in Fig. 3, which are included output signals and input signals. From Fig. 3, because of the fast time-varying disturbance (25), the close-loop control system cannot achieve asymptotic tracking under the [17]. However, it can be seen from Fig. 4, the tracking error significantly decreases using the proposed control method in this paper. The proposed model free controller can achieve a better performance in the presence of same fast timevarying disturbance (25). 
International Journal of Instrumentation and Control Systems (IJICS) Vol.5, No.4, October 2015
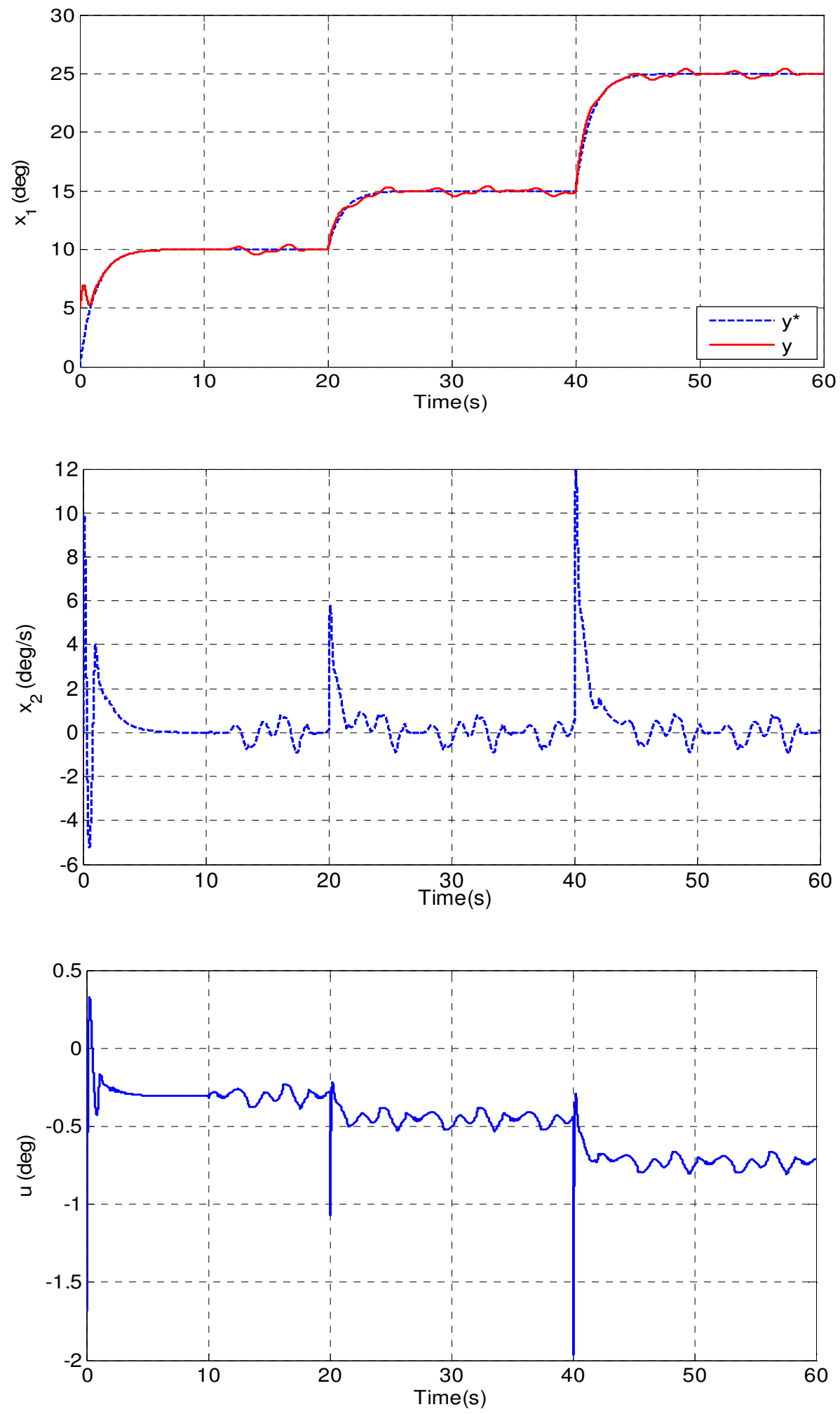

Figure 3: System responses using the control approach of [17] 
International Journal of Instrumentation and Control Systems (IJICS) Vol.5, No.4, October 2015
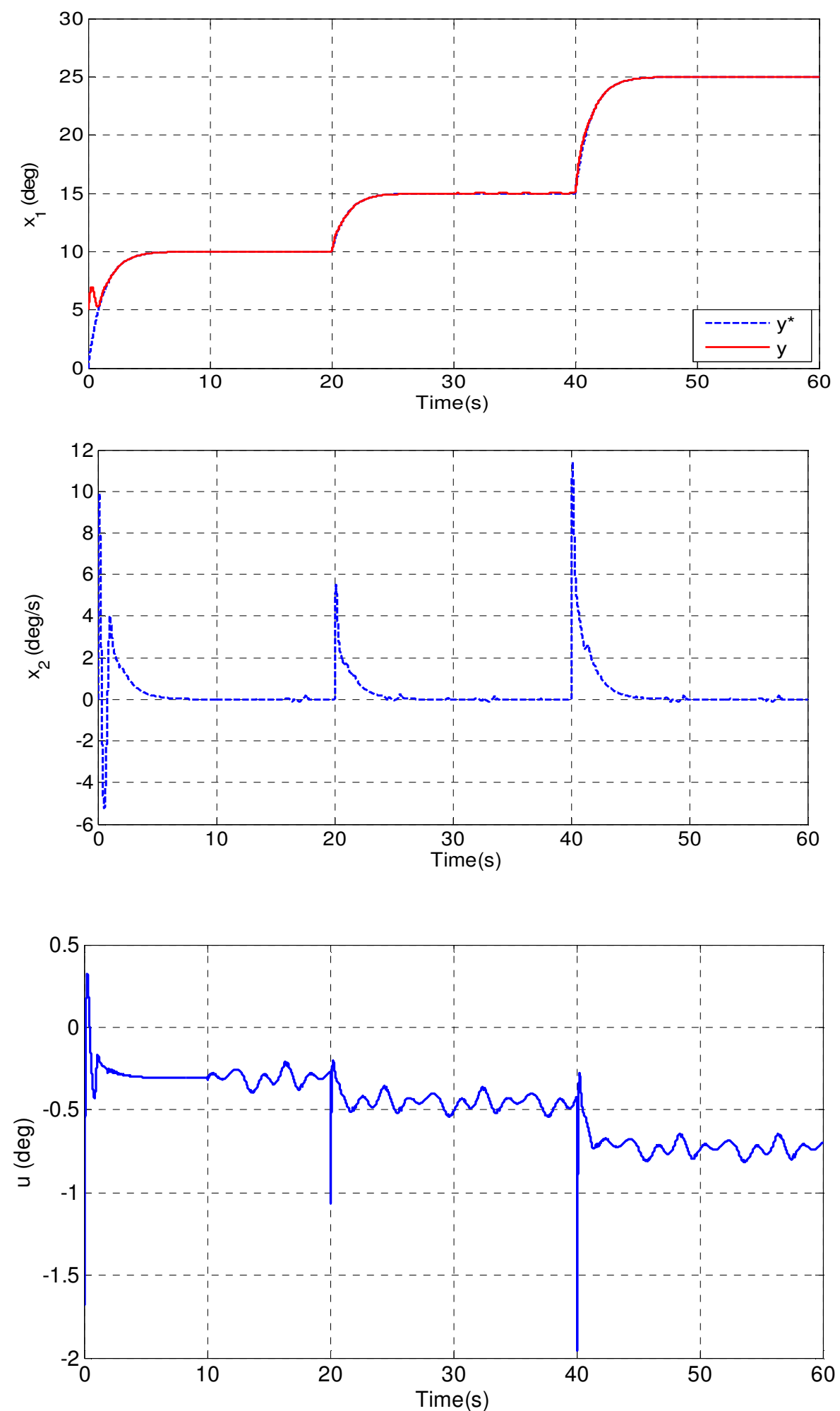

Figure 4: System responses using the proposed control approach in this paper. 


\section{Conclusions}

We have studied a systematic study on the yaw channel of a UAV helicopter in this paper. The yaw channel of an unmanned-aerial-vehicle helicopter is non-affine in the control input. In order to improve operational performance, we have developed a new MFAC algorithm via CFDL. The proposed MFAC scheme can guarantee the asymptotic output tracking of the closed-loop control systems in spite of unknown uncertainties and disturbances. Finally, simulation results are provided on yaw dynamics of a small-scale UAV helicopter to show the effective and advantages of the new proposed control strategy.

\section{ACKNOWLEDGEMENTS}

This work is supported by National Natural Science Foundation of China (61503156), the Fundamental Research Funds for the Central Universities (JUSRP11562).

\section{REFERENCES}

[1] I. Raptis, K. Valavanis. Linear and nonlinear control of small-scale unmanned helicopters. SpringerVerlag, Berlin, 2011.

[2] E. Johnson, S. Kannan. Adaptive trajectory control for autonomous helicopters. AIAA Journal of Guidance, Control, and Dynamics, 28(3), 524-538, 2005.

[3] H. Gao, T. Chen. Network based H1 output tracking control. IEEE Trans. on Automatic Control, 2008, 53(3): 655-667

[4] G. Cai, B. Chen and K. Peng, etc. Modeling and control of the yaw channel of a UAV helicopter. IEEE Trans. on Industrial Electronics, 55(9), 3426-3434, 2008.

[5] S. Pieper, J. Baillie and K. Goheen. Linear-quadratic optimal model-following control of a helicopter in hover. Optimal Control Applications and Methods, 17(2), 123-140, 1998.

[6] E. Prempain, I. Postlethwaite. Static loop shaping control of a fly-by-wire helicopter. Automatica, 41(9): 1517-1528, 2005.

[7] J. Shin, K. Nonami and D. Fujiwara, etc. Model-based optimal attitude and positioning control of smallscale unmanned helicopter. Robotica, 23, 51-63, 2005.

[8] H. Kim, H. Dharmayanda, T. Kang, A. Budiyono, G. Lee, and W. Adiprawita. Parameter identification and design of a robust attitude controller using methodology for the raptor E620 smallscale helicopter. International Journal of Control, Automation, and Systems. 10(1): 88-101, 2012.

[9] M. Weilenmann, U. Christen and H. Geering. Robust helicopter position control at hover. Proceedings of the American Control Conference, 2491-2495, 1994, Baltimore, MD.

[10] X. Zhao, J. Han. Yaw control of helicopter: an adaptive guaranteed cost control approach. International Journal of Innovative Computing, Information and Control, 5(8): 2267-2276, 2009.

[11] L. Marconi, R. Naldi. Robust full degree-of-freedom tracking control of a helicopter. Automatica, 43(11): 1909-1920, 2007.

[12] H. Wang, A. Mian, D. Wang, and H. Duan. Robust multi-mode flight control design for an unmanned helicopter based on multi-loop structure. International Journal of Control, Automation, and Systems. 7(5): 723-730, 2009.

[13] B. Song, J. Mills, Y. Liu, and C. Fan. Nonlinear dynamic modeling and control of a small-scale helicopter. International Journal of Control, Automation, and Systems. 8(3): 534-543, 2010.

[14] J. Boskovic, L. Chen and R. Mehra. Adaptive control design for nonaffine models arising in flight control. AIAA Journal of Guidance, Control, and Dynamics, 27(2): 209-217, 2004.

[15] Z. Hou, S. Jin, A novel data-driven control approach for a class of discrete-time nonlinear systems, IEEE Trans. on Control Systems Technology, 19(6), 1549-1558, 2011.

[16] D. Xu, B. Jiang, P. Shi, A novel model free adaptive control design for multivariable industrial processes. IEEE Transactions on Industrial Electronics, vol. 61, no. 11, pp. 6391-6398, 2014. 
International Journal of Instrumentation and Control Systems (IJICS) Vol.5, No.4, October 2015

[17] D. Xu, B. Jiang, P. Shi, Adaptive observer based data-driven control for nonlinear discrete-time processes, IEEE Transactions on Automation Science and Engineering, vol. 11, no. 4, pp. 1073-1045, 2014.

[18] J. Leishman. Principles of helicopter aerodynamics. Cambridge University Press, 2rd edition, Cambridge, 2002.

[19] J. Spooner, M. Maggiore, and R. Ordonez etc., Stable adaptive control and estimation for nonlinear systems, Wiley, New York, 2002. 